\title{
VARIATIONAL ANALYSIS OF NASH EQUILIBRIA FOR A MODEL OF TRAFFIC FLOW
}

\author{
BY \\ ALBERTO BRESSAN (Department of Mathematics, Penn State University, University Park, \\ Pennsylvania 16802), \\ CHEN JIE LIU (Department of Mathematics, Shanghai Jiao Tong University, Shanghai, 200240, \\ China), \\ WEN SHEN (Department of Mathematics, Penn State University, University Park, Pennsylvania \\ 16802), \\ AND
}

FANG YU (Department of Mathematics, Shanghai Jiao Tong University, Shanghai, 200240, China)

Dedicated to Constantine Dafermos in the occasion of his 70th birthday

\begin{abstract}
The paper is concerned with Nash equilibrium solutions for the LighthillWhitham model of traffic flow, where each driver chooses his own departure time in order to minimize the sum of a departure cost and an arrival cost. Estimates are provided on how much the Nash solution may change, depending on the cost functions and on the flux function of the conservation law. It is shown that this equilibrium solution can also be determined as a global minimizer for a functional $\Phi$, measuring the maximum total cost among all drivers, in a given traffic pattern. The last section of the paper introduces two evolution models, describing how the traffic pattern can change, day after day. It is assumed that each driver adjusts his departure time based on previous experience, in order to lower his own cost. Numerical simulations are reported, indicating a possible instability of the Nash equilibrium.
\end{abstract}

1. Introduction. In this paper we study properties of Nash equilibrium solutions for a model of traffic flow introduced in [1. Car drivers starting from a location $A$ (a residential neighborhood) need to reach a destination $B$ (a working place) all at a given time $T_{0}$. There is a cost $\varphi$ for starting early and a cost $\psi$ for arriving late. Denoting

Received November 16, 2011.

2010 Mathematics Subject Classification. Primary 35L65; Secondary 35Q93, 49J21, 49N70, 49N90.

E-mail address: bressan@math.psu.edu

E-mail address: cjliusjtu@gmail.com

E-mail address: shen_w@psu.edu

E-mail address: yufang0820@sjtu.edu.cn 
by $\tau_{d}$ and $\tau_{a}$ respectively the departure and the arrival time of an individual driver, his total cost is thus measured by

$$
\Psi \doteq \varphi\left(\tau_{d}\right)+\psi\left(\tau_{a}\right) .
$$

We assume that each driver can choose his departure time in order to minimize this total cost. A distribution of departure times is called a Nash equilibrium if no driver can reduce his total cost by choosing a different departure time. Of course, in this case all drivers will bear the same total cost.

It is assumed that all cars travel along the same highway. Calling $\rho=\rho(t, x)$ the density of cars at time $t$ at the point $x$ along the road, the Lighthill-Whitham model [9] describes the evolution of $\rho$ in terms of the conservation law

$$
\rho_{t}+[\rho v(\rho)]_{x}=0 .
$$

Here $v=v(\rho)$ is a decreasing function, determining how the velocity of cars varies with the density. In a typical setting (Fig. 1 left), the flux function $\rho \mapsto \rho v(\rho)$ is concave down and attains a maximum $M=\rho^{*} v\left(\rho^{*}\right)$ at some value $\rho^{*}$ of the density.

As remarked in [1, it is convenient to write (1.2) as a conservation law for the traffic flux $u=\rho v(\rho)$, namely

$$
u_{x}+f(u)_{t}=0 \text {. }
$$

Here we define $f(u)=\rho$ as a partial inverse of the original flux function (Fig 1 , center). More precisely, for $u \in[0, M]$ we set

$$
f(u)=\rho \quad \text { iff } \quad u=\rho v(\rho), \quad \rho \in\left[0, \rho^{*}\right] .
$$

Then we extend $f$ outside the interval $[0, M]$ by setting

$$
f(u) \doteq+\infty \quad \text { if } u<0 \text { or } u>M .
$$

The Legendre transform of this extended flux function (Fig. 1, right) will be denoted by

$$
f^{*}(p) \doteq \max _{u}\{p u-f(u)\}
$$
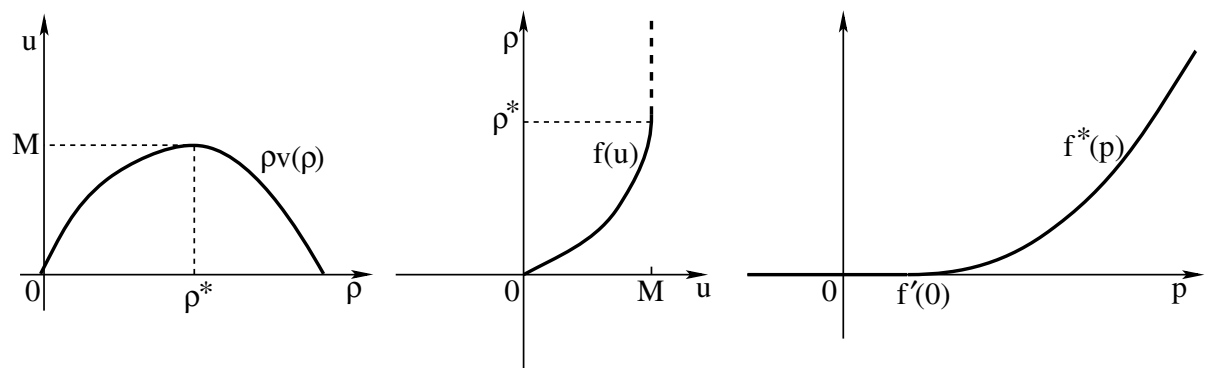

FIG. 1. Left: the function $\rho \mapsto \rho v(\rho)$ describing the flux of cars. Middle: the function $f$, implicitly defined by $f(\rho v(\rho))=\rho$ and extended according to (1.5). Right: the Legendre transform $f^{*}$.

Throughout the following, for notational convenience we shall invert the role of the independent variables $t, x$, and write (1.3) in the form

$$
u_{t}+f(u)_{x}=0 .
$$


Introducing the integral function

$$
U(t, x) \doteq \int_{-\infty}^{x} u(t, s) d s
$$

the conservation law (1.7) can be equivalently written as a Hamilton-Jacobi equation

$$
U_{t}+f\left(U_{x}\right)=0 .
$$

For our model of traffic flow, one should keep in mind that now the $x$ variable denotes time, while $t \in[0, T]$ denotes a specific point on a highway of total length $T$. Hence $U(t, x)$ measures the total number of cars that have crossed the point $t$ along the highway during the time interval $]-\infty, x]$.

As initial data for (1.8) we consider any bounded nondecreasing function $Q: \mathbb{R} \mapsto \mathbb{R}^{+}$, with

$$
Q(-\infty)=0, \quad Q(+\infty)=\kappa .
$$

Here $Q(x)$ denotes the total number of cars that have entered the queue at the entrance of the highway up to time $x$, while $\kappa$ is the total number of departing cars. Being monotone, the function $Q$ is continuous with the possible exception of countably many times $x$. To fix the ideas, we shall consider the left-continuous version where $Q(x)=Q(x-)$ coincides with its left limit at every $x$. When needed, we shall denote by $Q(x+)=\lim _{y \rightarrow x+} Q(y)$ the right limit of $Q$ at $x$.

For $t>0$, the entropy-admissible solution to the Cauchy problem (1.8) is provided by the Lax formula:

$$
U(t, x)=\min \left\{t f^{*}\left(\frac{x-y}{t}\right)+Q(y): y \in \mathbb{R}\right\} .
$$

Notice that, since $Q(\cdot)$ is nondecreasing and left continuous, the minimum in (1.10) is well defined. As shown in [1, one has

$$
U(0+, x)=\bar{U}(x) \doteq \min \{Q(y)+M(x-y): y \leq x\} \leq Q(x) .
$$

Indeed, there is a maximum flux $M$ of cars that can enter the highway. If $Q$ is discontinuous, or simply if $Q_{x}>M$, then a queue is formed at the entrance of the highway. The quantity $Q(x)-\bar{U}(x)$ measures the length of this queue at time $x$, while $\bar{U}(x)$ denotes the total number of drivers that have actually departed (after clearing the queue) up to time $x$. With the above notation, the map $x \mapsto U(T, x)$ describes how many cars have arrived at the destination, i.e. at the end of the highway, within time $x$.

Given a nondecreasing, left continuous initial data $Q(\cdot)$ as in (1.9]), for $\beta \in[0, \kappa[$ we define the times $x^{q}(\beta), x^{d}(\beta)$, and $x^{a}(\beta)$ by setting

$$
\left\{\begin{array}{l}
x^{q}(\beta)=\max \{x \in \mathbb{R}: Q(x) \leq \beta\}, \\
x^{d}(\beta)=\max \{x \in \mathbb{R}: U(0+, x) \leq \beta\}, \\
x^{a}(\beta)=\max \{x \in \mathbb{R}: U(T, x) \leq \beta\} .
\end{array}\right.
$$

Here $\beta$ is a Lagrangian variable labelling a particular driver, $x^{q}(\beta)$ accounts for the time where this driver joins the queue, $x^{d}(\beta)$ is the actual departure time (after clearing the 
queue), and $x^{a}(\beta)$ is the arrival time. Observe that, for all except countably many $\beta$, the above times are uniquely determined by the relations

$$
Q\left(x^{q}(\beta)\right) \leq \beta \leq Q\left(x^{q}(\beta)+\right), \quad \bar{U}\left(x^{d}(\beta)\right)=\beta, \quad U\left(T, x^{a}(\beta)\right)=\beta .
$$

More generally, if a driver starts at time $x$, to determine his arrival time we consider two possibilities.

- If there is no traffic at all, then the total time needed for the trip is

$$
\mu \doteq T f^{\prime}(0)=\frac{[\text { length of the highway }]}{[\text { maximum speed }]} .
$$

- On the other hand, if the driver starting at time $x$ encounters traffic, his arrival time will simply be the supremum among the arrival times of all cars that departed earlier.

Combining the above cases, the arrival time of a driver who starts at time $x$ is thus defined as

$$
A(x) \doteq \max \left\{x+\mu, \sup _{\beta<Q(x)} x^{a}(\beta)\right\} .
$$

As in [1, a Nash equilibrium solution can be defined as follows.

Definition. We say that a bounded, nondecreasing initial data $Q(\cdot)$ satisfying (1.9) yields a Nash solution of the Cauchy problem (1.8)-(1.11) with initial and terminal cost functions $\varphi, \psi$ if there exists a constant $c$ such that:

(i) For almost every $\beta \in[0, \kappa]$, one has

$$
\varphi\left(x^{q}(\beta)\right)+\psi\left(x^{a}(\beta)\right)=c .
$$

(ii) For all $x \in \mathbb{R}$, one has

$$
\varphi(x)+\psi(A(x)) \geq c .
$$

According to (i) all drivers bear the same cost $c$, while (ii) implies that no one can achieve a cost $<c$.

For a given total number $\kappa$ of drivers, the main result in [1] established the existence and uniqueness of a Nash equilibrium solution. The aim of this paper is to analyze the properties of this solution.

- In Section 2 we prove that the Nash equilibrium depends continuously on the cost functions $\varphi, \psi$ and on the speed of the cars $v=v(\rho)$. Assuming (without loss of generality) that the total cost to each driver is zero, our main results can be summarized as follows. Given a total number of drivers $\kappa$ and cost functions $\varphi_{i}, \psi_{i}, v_{i}, i=1,2$, the departure distributions $Q_{1}, Q_{2}$ of the corresponding Nash equilibria satisfy an estimate of the form

$$
\left\|Q_{1}-Q_{2}\right\|_{\mathbf{L}^{1}} \leq C \cdot\left(\left\|\varphi_{1}-\varphi_{2}\right\|_{\mathcal{C}^{0}}+\left\|\psi_{1}-\psi_{2}\right\|_{\mathcal{C}^{0}}+\left\|v_{1}-v_{2}\right\|_{\mathcal{C}^{0}}^{1 / 2}\right) .
$$

- Section 3 provides a minimax property of the Nash solution. Given any departure distribution $Q(\cdot)$, let $\Psi(Q)$ be the maximum cost among all drivers. For a fixed number of drivers, we show that the Nash equilibrium solution is a global minimizer of the functional $\Psi$. 
- In Section 4 we introduce two evolution equations, modeling how the departure distribution changes from one day to the next. In both cases, the Nash equilibrium corresponds to the unique steady state. The stability of this invariant configuration is a difficult issue, because the evolution equation contains nonlocal terms. Our numerical simulations suggest that the equilibrium configuration may be unstable, while the orbits approach a chaotic attractor.

For future reference, we state the basic assumptions that will be used, on the flux function $f$ in (1.4)-(1.5) and on the cost functions $\varphi, \psi$ in (1.1).

(A1) Restricted to the open interval $] 0, M[$, the flux function $f$ is twice continuously differentiable and satisfies

$$
\left.f^{\prime}(u)>0, \quad f^{\prime \prime}(u) \geq \gamma>0 \quad \forall u \in\right] 0, M[.
$$

Moreover, the following limits exist:

$$
\lim _{u \rightarrow 0+} f(u)=0, \quad \lim _{u \rightarrow 0+} f^{\prime}(u)>0, \quad \lim _{u \rightarrow M-} f^{\prime}(u)=+\infty, \quad \lim _{u \rightarrow 0+} f^{\prime \prime}(u)<+\infty .
$$

(A2) The cost functions $\varphi, \psi$ are continuously differentiable and satisfy

$$
\varphi^{\prime}<0, \quad \psi, \psi^{\prime} \geq 0, \quad \lim _{x \rightarrow-\infty} \varphi(x)=+\infty, \quad \lim _{x \rightarrow+\infty}(\varphi(x)+\psi(x))=+\infty .
$$

For a review of the extensive literature on mathematical models of traffic flow we refer to [4. See also [2, 3, 5, 6] for related optimization problems.

2. Continuous dependence. In this section we study how the Nash equilibrium changes, depending on the cost functions $\varphi, \psi$ and on the flux $f$. We always assume that (A1)-(A2) are satisfied.

Given a common cost $c$, for each $x \in \mathbb{R}$, let $\Lambda(x)$ be the point such that

$$
\varphi(\Lambda(x))+\psi(x)=c,
$$

with the provision that $\Lambda(x)=+\infty$ if $\psi(x) \geq c-\inf _{y \in \mathbb{R}} \varphi(y)$. Since $\varphi$ is strictly decreasing, $\Lambda(x)$ is uniquely defined.

REMARK 1. Recalling the definition of arrival time in (1.15), a Nash equilibrium solution $U$ of (1.8) with common cost $c$ to all drivers can be equivalently characterized by the two requirements:

(N1) $Q(\Lambda(x)) \leq U(T, x) \leq Q(\Lambda(x)+)$ for a.e. $x$ such that $U_{x}(T, x)>0$,

(N2) $x \leq A(\Lambda(x))$ for all $x \in \mathbb{R}$.

By this characterization, the Nash solution is entirely determined by the flux function $f$ and by the map $\Lambda$. We also observe that, by replacing $\varphi$ with $\varphi-c$, it is not restrictive to consider the special case where the common cost to all drivers is zero.

For a given flux function $f$, it will be convenient to introduce the function

$$
h(s) \doteq-T f^{*}\left(\frac{-s}{T}\right) ;
$$


see Fig. 2, left. Given an initial data $Q(\cdot)$, by (1.10) the terminal profile $U(T, \cdot)$ is computed by (see Fig. 2, right)

$$
U(T, x)=\min _{y \in \mathbb{R}}\{Q(y)-h(y-x)\} .
$$

We now review some identities satisfied by the Legendre transform. For each $p \in$ ]$f^{\prime}(0),+\infty[$, let $u(p)$ denote the point where the maximum in (1.6) is attained. Then one has

$$
f^{\prime}(u(p))=p, \quad f^{*}(p)=p u(p)-f(u(p)) .
$$

Differentiating the two identities in (2.4) w.r.t. $p$, one obtains

$$
f^{\prime \prime}(u(p)) u^{\prime}(p)=1, \quad\left(f^{*}\right)^{\prime}(p)=u(p), \quad\left(f^{*}\right)^{\prime \prime}(p)=u^{\prime}(p)=\frac{1}{f^{\prime \prime}(u(p))} .
$$

As in (1.14), let $\mu \doteq T f^{\prime}(0)$. We now write a second-order Taylor expansion of the function $h$ at the point $-\mu$, observing that $f^{*}\left(f^{\prime}(0)\right)=\left(f^{*}\right)^{\prime}\left(f^{\prime}(0)\right)=0$ (Fig. 1, right). By the assumptions (1.18)-(1.19), since $f^{\prime \prime}(u) \geq \gamma>0$, for every $s<-\mu$ one obtains

$$
h(s)=\frac{(s+\mu)^{2}}{2} h^{\prime \prime}\left(s_{1}\right)=-\frac{(s+\mu)^{2}}{2 T}\left(f^{*}\right)^{\prime \prime}\left(\frac{-s_{1}}{T}\right) \geq-\frac{(s+\mu)^{2}}{2 \gamma T},
$$

where $s_{1} \in[s,-\mu]$ is a suitable point. Using (2.3) and (2.6), for every $\varepsilon \geq 0$ we deduce

$$
U(T, x) \leq Q(x-\mu-\varepsilon)+\frac{\varepsilon^{2}}{2 \gamma T} .
$$

Given departure and arrival costs $\varphi, \psi$, as proved in [1], the departure distribution $Q^{*}(\cdot)$ for the Nash equilibrium solution, where all drivers bear the common $\operatorname{cost} c=0$, can be obtained as

$$
Q^{*}(x)=\sup \left\{Q(x) ; Q \in \mathcal{Q}_{0}\right\} .
$$

Here $\mathcal{Q}_{0}$ is the family of all starting distributions where each driver pays a cost $\leq 0$, i.e.

$$
\begin{aligned}
\mathcal{Q}_{0} & \doteq\left\{Q(\cdot): \varphi\left(x^{q}(\beta)\right)+\psi\left(x^{a}(\beta)\right) \leq 0 \quad \text { for a.e. } \beta \in[0, Q(+\infty)]\right\} \\
& =\left\{Q(\cdot): x^{q}(\beta) \geq \Lambda\left(x^{a}(\beta)\right) \quad \text { for a.e. } \beta \in[0, Q(+\infty)]\right\} .
\end{aligned}
$$

We recall that the starting time $x^{q}$ and the arrival time $x^{a}$ of the $\beta$-driver are defined as in (1.12). Of course, these depend on the overall traffic pattern, hence on the flux function $f$ as well as on $Q(\cdot)$.

Theorem 1 (comparison). Let $Q^{*}(\cdot)$ be the initial data for the Nash equilibrium solution (with zero total cost to each driver), corresponding to the departure and arrival costs $\varphi, \psi$ and to the flux function $f$. Similarly, let $\widetilde{Q}(\cdot)$ be the initial data for the Nash solution given the costs $\tilde{\varphi}, \tilde{\psi}$ and the flux function $\tilde{f}$. As in (2.1), let $\Lambda, \widetilde{\Lambda}$ be implicitly defined by

$$
\varphi(\Lambda(x))+\psi(x)=0, \quad \tilde{\varphi}(\widetilde{\Lambda}(x))+\tilde{\psi}(x)=0 .
$$

If

$$
\begin{aligned}
\Lambda(x) \leq \tilde{\Lambda}(x) & & \forall x \in \mathbb{R}, \\
f(u) \leq \tilde{f}(u) & & \forall u \geq 0,
\end{aligned}
$$


then

$$
\widetilde{Q}(x) \leq Q^{*}(x) \quad \forall x \in \mathbb{R}
$$

Proof. Thanks to (2.8), it suffices to show that $\widetilde{Q}(\cdot) \in \mathcal{Q}_{0}$. Let $h(s)$ and $\tilde{h}(s)$ be the functions defined in (2.2) , corresponding to $f$ and $\tilde{f}$ respectively. For the initial data $\widetilde{Q}(x)$, the terminal profile $\widetilde{U}(T, x)$ with the flux $\tilde{f}$ is computed by

$$
\widetilde{U}(T, x)=\min _{y \in \mathbb{R}}\{\widetilde{Q}(y)-\tilde{h}(y-x)\} .
$$

As in (1.12), we shall denote by $\tilde{x}^{q}(\beta)$ and $\tilde{x}^{a}(\beta)$ the starting time and the arrival time of the driver labelled by $\beta \in[0, \widetilde{Q}(+\infty)]$.

On the other hand, the terminal profile $\widehat{U}(T, x)$ for the same initial data $\widetilde{Q}(x)$ but with flux $f$ is computed by

$$
\widehat{U}(T, x)=\min _{y \in \mathbb{R}}\{\widetilde{Q}(y)-h(y-x)\} .
$$

In this case, the starting time and the arrival time for the driver $\beta \in[0, \widetilde{Q}(+\infty)]$, will be denoted by $\hat{x}^{q}(\beta), \hat{x}^{a}(\beta)$, respectively.

Using (1.6), (2.2), and (2.12), we obtain $h(s) \leq \tilde{h}(s)$. Hence $\widetilde{U}(T, x) \leq \widehat{U}(T, x)$ for all $x \in \mathbb{R}$. From (1.12) it thus follows that

$$
\hat{x}^{q}(\beta)=\tilde{x}^{q}(\beta), \quad \hat{x}^{a}(\beta) \leq \tilde{x}^{a}(\beta) \quad \text { for a.e. } \beta \in[0, \widetilde{Q}(+\infty)] .
$$

Since $\widetilde{Q}(\cdot)$ is the Nash equilibrium solution given the $\operatorname{costs} \tilde{\varphi}, \tilde{\psi}$ and the flux function $\tilde{f}$, for almost every $\beta$ one has the identity $\tilde{x}^{q}(\beta)=\widetilde{\Lambda}\left(\tilde{x}^{a}(\beta)\right)$. Using (2.11) and the monotonicity of the map $x \mapsto \widetilde{\Lambda}(x)$, for the initial data $\widetilde{Q}$ we obtain

$$
\hat{x}^{q}(\beta)=\tilde{x}^{q}(\beta)=\widetilde{\Lambda}\left(\tilde{x}^{a}(\beta)\right) \geq \widetilde{\Lambda}\left(\hat{x}^{a}(\beta)\right) \geq \Lambda\left(\hat{x}^{a}(\beta)\right) \quad \text { for a.e. } \beta \in[0, \widetilde{Q}(+\infty)] .
$$

Therefore $\widetilde{Q}(\cdot) \in \mathcal{Q}_{0}$ and $\widetilde{Q}(x) \leq Q^{*}(x)$ for all $x \in \mathbb{R}$.

REMARK 2. Since $\varphi, \tilde{\varphi}$ are strictly decreasing, the inequality (2.11) trivially holds if $\varphi \leq \tilde{\varphi}$ and $\psi \leq \tilde{\psi}$. Moreover, (2.12) holds in the case where $f, \tilde{f}$ are defined as in (1.4), for speeds $\tilde{v}(\rho) \leq v(\rho)$. In this case, Theorem 1 yields an intuitively obvious fact: by increasing the departure and arrival costs and decreasing the speed of the cars, the total number of cars in a Nash equilibrium solution (where every driver still pays zero cost) will decrease.

2.1. Continuous dependence on the cost functions. Next, we wish to relate two Nash equilibrium solutions with different cost and flux functions, without the assumptions (2.11) -(2.12). The first result describes how the Nash equilibrium changes when the cost functions $\varphi, \psi$ are changed.

Theorem 2. Let the flux function $f$ and the cost functions $\varphi_{i}, \psi_{i}, i=1,2$ satisfy the assumptions (A1)-(A2). Let $Q_{1}, Q_{2}$ be the starting distributions of the corresponding Nash equilibria. Assume that all drivers in both cases start and arrive within a time interval $I=[a, b]$. Then there exists a constant $C$ not depending on $Q_{1}, Q_{2}$ such that

$$
\left\|Q_{1}-Q_{2}\right\|_{\mathbf{L}^{1}([a, b])} \leq C \cdot\left\|\Lambda_{1}-\Lambda_{2}\right\|_{\mathbf{L}^{\infty}([a, b])} .
$$



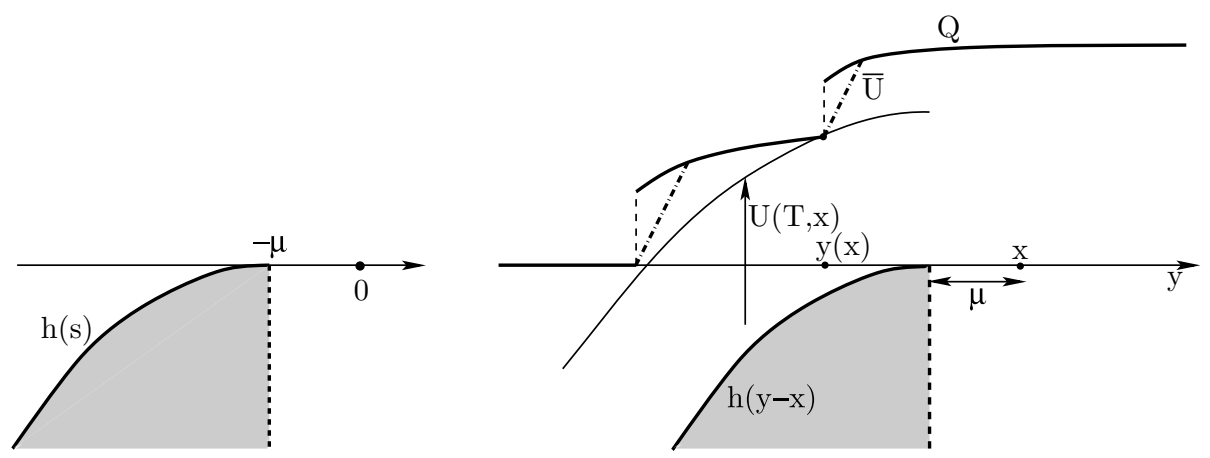

FIG. 2. Left: the graph of the function $h(\cdot)$ introduced in 2.2 . Right: by the Lax formula, $U(T, x)$ is the amount by which one can push up the graph of $h(\cdot-x)$ until it touches the graph of $Q(\cdot)$. Clearly, $U(T, x) \leq Q(x-\mu)$.

Proof. Let

$$
\varepsilon \doteq \sup _{x \in[a, b]}\left|\Lambda_{1}(x)-\Lambda_{2}(x)\right|
$$

It is clearly not restrictive to assume $0<\varepsilon \leq 1$.

1. Let any $\bar{x} \in[a, b]$ be given. Our goal is to prove an estimate of the form

$$
Q_{2}\left(\Lambda_{1}(\bar{x})-\varepsilon\right)-Q_{1}\left(\Lambda_{1}(\bar{x})\right) \leq C_{0} \varepsilon
$$

for some constant $C_{0}$. For this purpose, we construct two decreasing sequences

$$
\bar{x}=x_{0}>x_{1}>\cdots>x_{n}, \quad y_{k}=\Lambda_{1}\left(x_{k}\right),
$$

according to the following inductive rule. Given $x_{k}$, consider two cases:

CASE 1. $y_{k} \leq x_{k}-\mu-\varepsilon$. In this case, we choose $y_{k+1}$ to be the smallest point where the minimum defining $U_{1}\left(T, x_{k}\right)$ is attained. More precisely:

$$
y_{k+1} \doteq \min \left\{\tilde{y}: Q_{1}(\tilde{y})-h\left(\tilde{y}-x_{k}\right)=\min _{y \leq x_{k}}\left\{Q_{1}(y)-h\left(y-x_{k}\right)\right\}\right\} .
$$

We then set

$$
x_{k+1} \doteq \min \left\{x: \Lambda_{1}(x)=y_{k+1}\right\} .
$$

CASE 2. $y_{k}>x_{k}-\mu-\varepsilon$. In this case we set $y_{k+1} \doteq y_{k}-\varepsilon$ and define $x_{k+1}$ as in (2.18).

The construction terminates when $y_{n} \leq a$ for some $n \geq 1$. Observe that, for some constant $c_{0}>0$ suitably small, one has

either $\quad Q_{1}\left(y_{k+1}\right) \leq Q_{1}\left(y_{k}\right)+h(-\mu-\varepsilon) \leq Q_{1}\left(y_{k}\right)-c_{0} \varepsilon^{2} \quad$ or $\quad y_{k+1} \leq y_{k}-\varepsilon$.

Therefore the induction must terminate after a finite number of steps. 

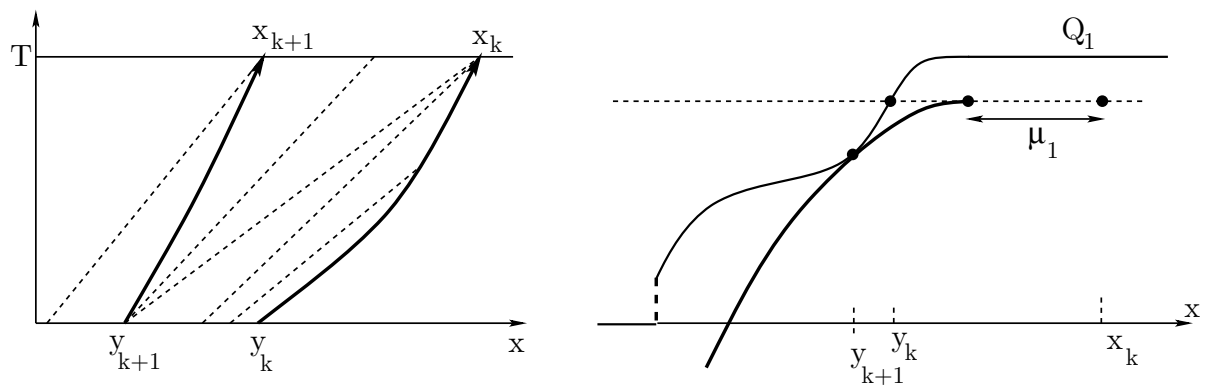

FIG. 3. Construction of the points $x_{k}, y_{k}$ in Case 1. Here the thick solid lines denote car trajectories, while the dotted lines denote characteristics. The point $x_{k}$ is the arrival time of the driver who starts at time $y_{k}=\Lambda_{1}\left(x_{k}\right)$. Moreover, $y_{k+1}$ is the initial point of the characteristic through the point $\left(T, x_{k}\right)$. If several characteristics reach the same point $x_{k}$ (this can happen if $x_{k}$ is the location of a shock), then we choose $y_{k+1}$ on the minimal backward characteristic.

2. In Case 1 , since $y_{k+1}=\Lambda_{1}\left(x_{k+1}\right)$ and $h^{\prime \prime} \leq 0$, our construction yields

$$
\begin{aligned}
Q_{2}( & \left.\Lambda_{1}\left(x_{k}\right)-\varepsilon\right)-Q_{1}\left(\Lambda_{1}\left(x_{k}\right)\right) \\
& \leq\left[Q_{2}\left(y_{k+1}-\varepsilon\right)-h\left(y_{k+1}-\varepsilon-x_{k}\right)\right]-\left[Q_{1}\left(y_{k+1}\right)-h\left(y_{k+1}-x_{k}\right)\right] \\
& \leq\left[Q_{2}\left(\Lambda_{1}\left(x_{k+1}\right)-\varepsilon\right)-Q_{1}\left(\Lambda_{1}\left(x_{k+1}\right)\right)\right]+h^{\prime}\left(y_{k+1}-\varepsilon-x_{k}\right) \cdot \varepsilon .
\end{aligned}
$$

In Case 2, by (2.6) our construction yields

$$
Q_{2}(y)-h\left(y-x_{k}\right) \geq U_{2}\left(T, x_{k}\right)=Q_{2}\left(\Lambda_{2}\left(x_{k}\right)\right) \geq Q_{2}\left(\Lambda_{1}\left(x_{k}\right)-\varepsilon\right)
$$

for all $y \leq x_{k}$. Since $y_{k}-x_{k}>-\mu-\varepsilon$, taking $y=y_{k+1}-\varepsilon=y_{k}-2 \varepsilon$ we obtain

$$
\begin{aligned}
Q_{2}\left(y_{k}-\varepsilon\right) & \leq Q_{2}\left(y_{k+1}-\varepsilon\right)-h\left(y_{k+1}-\varepsilon-x_{k}\right) \\
& \leq Q_{2}\left(y_{k+1}-\varepsilon\right)-h(-\mu-2 \varepsilon) \leq Q_{2}\left(y_{k+1}-\varepsilon\right)+\frac{4 \varepsilon^{2}}{2 \gamma T}, \\
Q_{2}\left(y_{k}-\varepsilon\right)-Q_{1}\left(y_{k}\right) & \leq Q_{2}\left(y_{k+1}-\varepsilon\right)+\frac{2 \varepsilon^{2}}{\gamma T}-Q_{1}\left(y_{k+1}\right) .
\end{aligned}
$$

Since $y_{n} \leq a$, we have $Q_{2}\left(y_{n}-\varepsilon\right)=Q_{1}\left(y_{n}\right)=0$. Splitting the set of indices $\mathcal{I}=$ $\{1, \ldots, n\}=\mathcal{I}_{1} \cup \mathcal{I}_{2}$ according to whether Case 1 or Case 2 applies, we obtain

$$
\begin{aligned}
& Q_{2}\left(\Lambda_{1}(\bar{x})-\varepsilon\right)-Q_{1}\left(\Lambda_{1}(\bar{x})\right)=Q_{2}\left(y_{0}-\varepsilon\right)-Q_{1}\left(y_{0}\right) \\
& \quad \leq \varepsilon \cdot \sum_{k \in \mathcal{I}_{1}} h^{\prime}\left(y_{k+1}-\varepsilon-x_{k}\right)+\sum_{k \in \mathcal{I}_{2}} \frac{2 \varepsilon^{2}}{\gamma T} \doteq J_{1}+J_{2} .
\end{aligned}
$$

3. Since the total number of steps where Case 2 can occur is $\leq(b-a) / \varepsilon$, the second summation in (2.21) is estimated by

$$
J_{2} \leq \frac{2 \varepsilon^{2}}{\gamma T} \cdot \frac{b-a}{\varepsilon}=\frac{2(b-a)}{\gamma T} \varepsilon .
$$


We now work toward an estimate of $J_{1}$. By the properties of the function $h$ in (2.2), there exists a constant $C_{1}$ such that

$$
h^{\prime}(s) \leq C_{1}(-\mu-s) \quad \forall s \in[a-b,-\mu] .
$$

Define the constant

$$
M_{1} \doteq \inf _{a-b<s<-\mu} \frac{-h(s)}{(s+\mu)^{2}}>0
$$

and choose a suitably small constant $0<c_{2}<M_{1} /\left(3 C_{1}\right)$.

Two possibilities need to be considered. If

$$
y_{k}-y_{k+1} \geq c_{2}\left(x_{k}-\mu-y_{k+1}\right),
$$

observing that in Case 1 we have

$$
\varepsilon \leq x_{k}-\mu-y_{k} \leq x_{k}-\mu-y_{k+1},
$$

we conclude

$$
h^{\prime}\left(y_{k+1}-\varepsilon-x_{k}\right) \leq C_{1}\left(-\mu-y_{k+1}+\varepsilon+x_{k}\right) \leq \frac{2 C_{1}}{c_{2}}\left(y_{k}-y_{k+1}\right) .
$$

On the other hand, if (2.25) fails, then we claim that either $k=0$ or else

$$
h^{\prime}\left(y_{k+1}-\varepsilon-x_{k}\right) \leq C_{3}\left(x_{k-1}-x_{k}\right) .
$$

Indeed, if $k \geq 1$, at the previous iteration one has (see Fig. 44)

$$
\begin{aligned}
Q_{1}\left(y_{k-1}\right) & =Q_{1}\left(y_{k}\right)-h\left(y_{k}-x_{k-1}\right)=\min _{y}\left\{Q_{1}(y)-h\left(y-x_{k-1}\right)\right\} \\
& \leq Q_{1}\left(y_{k+1}\right)-h\left(y_{k+1}-x_{k-1}\right), \\
Q_{1}\left(y_{k}\right)-Q_{1}\left(y_{k+1}\right) & =-h\left(y_{k+1}-x_{k}\right) \leq h\left(y_{k}-x_{k-1}\right)-h\left(y_{k+1}-x_{k-1}\right) \\
& \leq h^{\prime}\left(y_{k+1}-x_{k-1}\right)\left(y_{k}-y_{k+1}\right) \\
& \leq C_{1}\left(x_{k-1}-\mu-y_{k+1}\right) \cdot c_{2}\left(x_{k}-\mu-y_{k+1}\right) .
\end{aligned}
$$

Using (2.24) and recalling that $c_{2} \leq M_{1} /\left(3 C_{1}\right)$, we obtain

$$
x_{k-1}-\mu-y_{k+1} \geq \frac{-h\left(y_{k+1}-x_{k}\right)}{C_{1} c_{2}\left(x_{k}-\mu-y_{k+1}\right)} \geq \frac{M_{1}\left(x_{k}-\mu-y_{k+1}\right)^{2}}{C_{1} c_{2}\left(x_{k}-\mu-y_{k+1}\right)} \geq 3\left(x_{k}-\mu-y_{k-1}\right) \text {. }
$$

We can thus write

$$
x_{k-1}-x_{k}=\left(x_{k-1}-\mu-y_{k+1}\right)-x_{k}+\mu+y_{k+1} \geq 2\left(x_{k}-\mu-y_{k+1}\right) .
$$

On the other hand, by (2.23) one has

$$
h^{\prime}\left(y_{k+1}-\varepsilon-x_{k}\right) \leq C_{1}\left(-\mu-y_{k+1}+\varepsilon+x_{k}\right) \leq 2 C_{1}\left(x_{k}-\mu-y_{k+1}\right),
$$

which yields (2.27).

Together, (2.26)-(2.27) yield the estimate

$$
\sum_{k} h^{\prime}\left(y_{k+1}-\varepsilon-x_{k}\right) \leq \sum_{k}\left[\frac{2 C_{1}}{c_{2}}\left(y_{k}-y_{k+1}\right)+C_{3}\left(x_{k}-x_{k+1}\right)\right] \leq\left(\frac{2 C_{1}}{c_{2}}+C_{3}\right)(b-a) \text {. }
$$




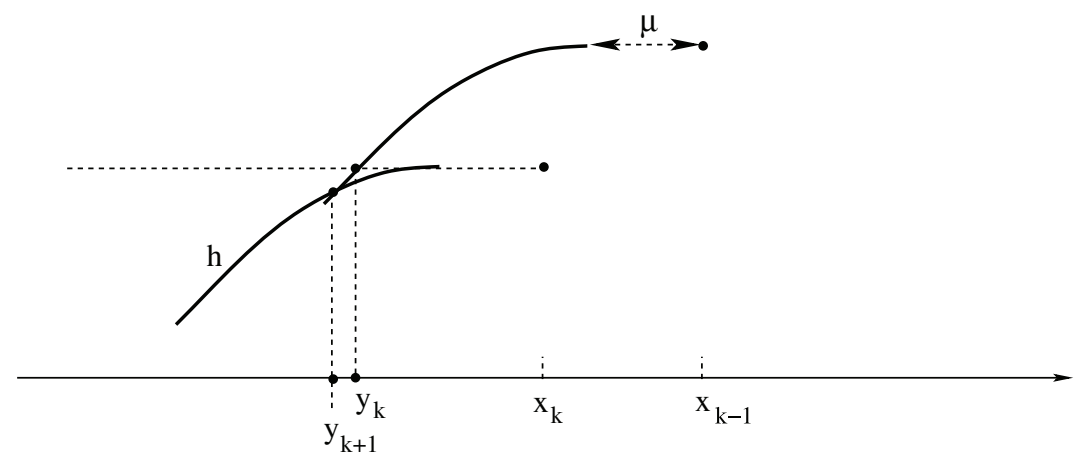

FIG. 4. If the difference $y_{k}-y_{k+1}$ is small compared with $x_{k}-\mu-$ $y_{k+1}$, then at the previous iteration the slope $h^{\prime}\left(y-x_{k-1}\right)$ must be much larger, on the interval $\left[y_{k+1}, y_{k}\right]$. Therefore $x_{k-1}-x_{k}$ must be large.

4. Using (2.22) and (2.30) in (2.21), we conclude that there exists a constant $C_{4}$ such that

$$
Q_{2}(y-\varepsilon)-Q_{1}(y) \leq C_{4}(b-a) \varepsilon \quad \forall y \in[a, b] .
$$

Inverting the roles of $Q_{1}, Q_{2}$ one obtains the similar inequality

$$
Q_{1}(y-\varepsilon)-Q_{2}(y) \leq C_{4}(b-a) \varepsilon \quad \forall y \in[a, b] .
$$

Denoting by $\kappa$ an upper bound for both functions $Q_{1}, Q_{2}$, we thus conclude that

$$
\begin{aligned}
\left\|Q_{2}-Q_{1}\right\|_{\mathbf{L}^{1}([a, b])}= & \int_{a}^{b}\left|Q_{2}(y)-Q_{1}(y)\right| d y \\
\leq & \int_{a}^{b}\left[Q_{2}(y-\varepsilon)-Q_{1}(y)\right]_{+} d y+\int_{a}^{b}\left[Q_{1}(y-\varepsilon)-Q_{2}(y)\right]_{+} d y \\
& +\int_{a}^{b}\left|Q_{1}(y)-Q_{1}(y-\varepsilon)\right| d y+\int_{a}^{b}\left|Q_{2}(y)-Q_{2}(y-\varepsilon)\right| d y \\
\leq & 2 C_{4}(b-a)^{2} \varepsilon+2 \kappa \varepsilon .
\end{aligned}
$$

2.2. Continuous dependence on the flux function. The main goal of this subsection is to understand how the Nash equilibrium solution changes if the velocity function $v=v(\rho)$ in (1.2) is changed. Our main result will be formulated in terms of the inverse of the function $h$ in (2.2). More precisely, given a flux function $f$ satisfying the assumptions (A1), for $y \leq 0$ we define

$$
h^{-1}(y) \doteq \min \left\{s \leq 0:-T f^{*}\left(\frac{-s}{T}\right) \geq y\right\} .
$$

TheOrem 3. Fix $\kappa>0$ and let the cost functions $\varphi$ and $\psi$ satisfy the assumptions (A2). Let $f_{1}, f_{2}$ be two flux functions, both satisfying (A1), and let $Q_{1}, Q_{2}$ be the starting distributions of the Nash equilibria with flux functions $f_{1}, f_{2}$, respectively. Assume that in both cases the total number of drivers is $\kappa$, and every driver starts and arrives within 
a time interval $I=[a, b]$, and has zero total cost. Then there exists a constant $C$ not depending on $Q_{1}, Q_{2}$ such that

$$
\left\|Q_{1}-Q_{2}\right\|_{\mathbf{L}^{1}([a, b])} \leq C \cdot\left\|h_{1}^{-1}-h_{2}^{-1}\right\|_{L^{\infty}([-\kappa, 0])} \cdot
$$

Proof. The estimate (2.34) will be derived from the bound (2.14) stated in Theorem 2.

1. Consider the map $x \mapsto \Lambda_{2}(x)$, implicitly defined by

$$
\varphi\left(\Lambda_{2}(x)\right)+\psi(x)=0 .
$$

By assumption, the initial datum $Q_{2}$ yields a Nash equilibrium solution in connection with the flux $f_{2}$, determined by the map $\Lambda_{2}(\cdot)$. We claim that $Q_{2}$ also yields a Nash solution in connection with the flux $f_{1}$, for some different costs $\tilde{\varphi}, \tilde{\psi}$, and a corresponding $\operatorname{map} \Lambda_{1}(\cdot)$.

Indeed, call $\kappa_{2} \doteq Q_{2}(+\infty)$. For every $\beta \in\left[0, \kappa_{2}\right]$ let $x_{1}^{a}(\beta)$ be the arrival time of the $\beta$-driver if the starting distribution is $Q_{2}$ and the flux function is $f_{1}$. As in (2.2), consider the functions

$$
h_{i}(s) \doteq-T f_{i}^{*}\left(\frac{-s}{T}\right), \quad i=1,2,
$$

where $f_{i}^{*}$ is the Legendre transform of $f_{i}$. We define the inverse functions $h_{1}^{-1}, h_{2}^{-1}$ by setting

$$
h_{i}^{-1}(w) \doteq \min \left\{s: h_{i}(s) \geq w\right\} \quad \forall w \leq 0 .
$$

Using (2.2) and (2.3), we obtain the representation

$$
x_{1}^{a}(\beta)=\sup _{\xi<\beta}\left\{x^{q}(\xi)-h_{1}^{-1}(\xi-\beta)\right\} .
$$

Observe that, in connection with the same initial distribution $Q_{2}$, if the flux function is $f_{2}$, then the arrival time is

$$
x_{2}^{a}(\beta)=\sup _{\xi<\beta}\left\{x^{q}(\xi)-h_{2}^{-1}(\xi-\beta)\right\} .
$$

From (2.37) and (2.38) it follows that

$$
\sup _{\beta \in\left[0, \kappa_{2}\right]}\left|x_{1}^{a}(\beta)-x_{2}^{a}(\beta)\right| \leq \sup _{\xi \in\left[-\kappa_{2}, 0\right]}\left|h_{1}^{-1}(\xi)-h_{2}^{-1}(\xi)\right| .
$$

2. For a.e. $\beta \in\left[0, \kappa_{2}\right]$, by assumption we have

$$
\varphi\left(\Lambda_{2}\left(x_{2}^{a}(\beta)\right)\right)+\psi\left(x_{2}^{a}(\beta)\right)=0 .
$$

We seek new cost functions $\tilde{\varphi}, \tilde{\psi}$, and a map $x \mapsto \Lambda_{1}(x)$ such that

$$
\tilde{\varphi}\left(\Lambda_{1}\left(x_{1}^{a}(\beta)\right)\right)+\tilde{\psi}\left(x_{1}^{a}(\beta)\right)=0 .
$$

Since we are assuming that all drivers depart and arrive within a given time interval $[a, b]$, using (2.39) and the identities

$$
\Lambda_{1}\left(x_{1}^{a}(\beta)\right)=x_{1}^{q}(\beta)=x_{2}^{q}(\beta)=\Lambda_{2}\left(x_{2}^{a}(\beta)\right),
$$

the difference $\Lambda_{1}-\Lambda_{2}$ can be estimated by

$$
\sup _{x \in[a, b]}\left|\Lambda_{1}(x)-\Lambda_{2}(x)\right| \leq\left(\inf _{x \in[a, b]} \frac{\partial}{\partial x} \Lambda_{1}(x)\right)^{-1} \cdot\left(\sup _{\beta \in\left[0, \kappa_{2}\right]}\left|x_{1}^{a}(\beta)-x_{2}^{a}(\beta)\right|\right),
$$


or equivalently by

$$
\sup _{x \in[a, b]}\left|\Lambda_{1}(x)-\Lambda_{2}(x)\right| \leq\left(\sup _{x \in[a, b]} \psi^{\prime}(x)\right) \cdot\left(\inf _{y \in[a, b]} \varphi^{\prime}(y)\right)^{-1} \cdot\left(\sup _{\beta \in\left[0, \kappa_{2}\right]}\left|x_{1}^{a}(\beta)-x_{2}^{a}(\beta)\right|\right) .
$$

The first two factors on the right-hand side of (2.42) depend only on the cost functions $\varphi, \psi$. The third factor is estimated by (2.39). Using Theorem 2 in connection with the flux function $f_{2}$ and the two maps $\Lambda_{1}, \Lambda_{2}$, we thus obtain

$$
\left\|Q_{1}-Q_{2}\right\|_{\mathbf{L}^{1}([a, b])} \leq C \cdot \sup _{\xi \in\left[-\kappa_{2}, 0\right]}\left|h_{1}^{-1}(\xi)-h_{2}^{-1}(\xi)\right|
$$
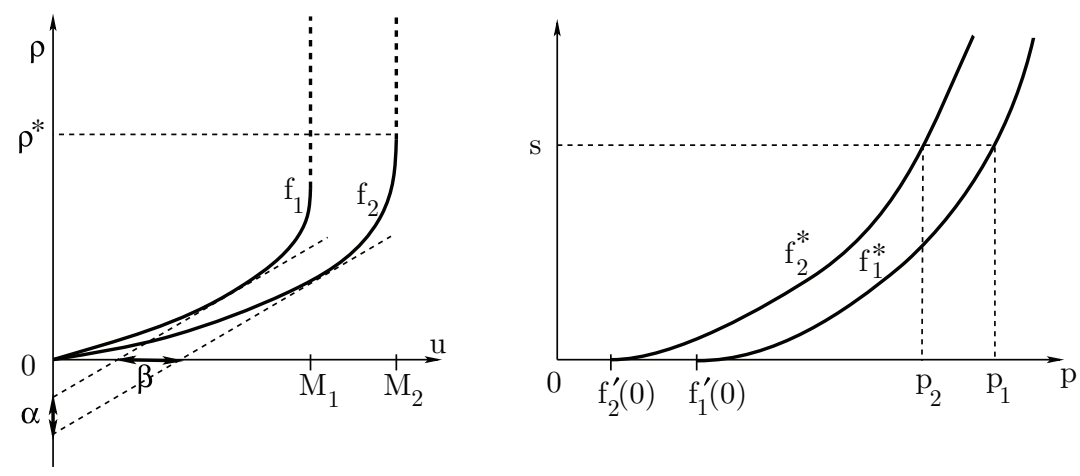

FIG. 5. Comparing two flux functions $f_{1}, f_{2}$ and their corresponding Legendre transforms.

To make use of the above theorem, one needs to understand how the distance $\| h_{1}^{-1}$ $h_{2}^{-1} \|_{\mathbf{L}^{\infty}([-\kappa, 0])}$ between the two inverse functions can be related to the difference between the flux functions $f_{1}, f_{2}$ and, in turn, with the difference between the velocity functions $v_{1}, v_{2}$ in (1.2). Up to a linear rescaling of coordinates, it is not restrictive to assume $T=1$. In the following, we thus seek to relate the distance $\left\|v_{1}-v_{2}\right\|_{\mathbf{L}}{ }$ with the distance $\left\|\left(f_{1}^{*}\right)^{-1}-\left(f_{2}^{*}\right)^{-1}\right\|_{\mathbf{L}^{\infty}}$ between the inverses of the Legendre transforms.

Let $v_{1}, v_{2}$ be smooth, nonincreasing velocity functions such that

$$
\frac{\partial^{2}}{\partial \rho^{2}} \rho v_{i}(\rho) \leq-\eta_{0}<0, \quad i=1,2 .
$$

Call $\rho_{i}^{*}$ the value where the flux function $\rho \mapsto \rho v_{i}(\rho)$ attains its global maximum, and let $\rho^{*} \doteq \max \left\{\rho_{1}^{*}, \rho_{2}^{*}\right\}$. Moreover, consider the distance

$$
\delta \doteq\left\|v_{1}-v_{2}\right\|_{\mathbf{L}^{\infty}\left(\left[0, \rho^{*}\right]\right)} .
$$

Let $\kappa>0$ be given. For any $s \in[0, \kappa]$, assume that the Legendre transforms of the corresponding functions $f_{1}, f_{2}$ in (1.4)-(1.5) satisfy

$$
f_{1}^{*}\left(p_{1}\right)=f_{2}^{*}\left(p_{2}\right)=s .
$$

We seek an estimate on $\left|p_{1}-p_{2}\right|$. 
Consider any slope $p \geq p_{0} \doteq \max \left\{f_{1}^{\prime}(0), f_{2}^{\prime}(0)\right\}$. Draw the lines with slope $p$ tangent to the graphs of $f_{1}, f_{2}$. Call $\alpha$ the distance between the interceptions of these lines with the $\rho$-axis, and $\beta$ the distance between the interceptions with the $u$-axis (see Fig. [5). Then

$$
\alpha=\left|f_{1}^{*}(p)-f_{2}^{*}(p)\right|=p \beta .
$$

Moreover, $\beta=\left|g_{1}^{*}(1 / p)-g_{2}^{*}(1 / p)\right|$, where

$$
g_{i}^{*}(q) \doteq \min _{\rho}\left\{q \cdot \rho-\rho v_{i}(\rho)\right\} .
$$

Given any $\bar{p}>p_{0}>0$, for all $p \in\left[p_{0}, \bar{p}\right]$ we thus have

$$
\left\|f_{1}^{*}-f_{2}^{*}\right\|_{\mathbf{L}^{\infty}([0, \bar{p}])} \leq \bar{p} \cdot \sup _{\rho \in\left[0, \rho^{*}\right]}\left|\rho v_{1}(\rho)-\rho v_{2}(\rho)\right| \leq \bar{p} \rho^{*}\left\|v_{1}-v_{2}\right\|_{\mathbf{L}^{\infty}\left(\left[0, \rho^{*}\right]\right)} .
$$

Recalling (2.4)-(2.5), consider the minimum of the second derivative

$$
\gamma_{i} \doteq \min _{p \in\left[p_{0}, \bar{p}\right]}\left(f_{i}^{*}\right)^{\prime \prime}(p)=\left(\max _{u \in\left[0, u_{i}(\bar{p})\right]} f_{i}^{\prime \prime}(u)\right)^{-1} .
$$

Set $\eta \doteq \min \left\{\gamma_{1}, \gamma_{2}\right\}$. If (2.45) holds with $s=0$, then

$$
\left|p_{1}-p_{2}\right|=\left|f_{1}^{\prime}(0)-f_{2}^{\prime}(0)\right|=\left|\frac{1}{v_{1}(0)}-\frac{1}{v_{2}(0)}\right| .
$$

More generally, for $s \in[0, \kappa]$ one has the estimate

$$
f_{2}^{*}\left(p_{1}+\left|f_{1}^{\prime}(0)-f_{2}^{\prime}(0)\right|+\delta\right) \geq f_{2}^{*}\left(p_{1}\right)+\frac{\eta}{2} \delta^{2} \geq f_{1}^{*}\left(p_{1}\right)-\left\|f_{1}^{*}-f_{2}^{*}\right\|_{\mathbf{L}^{\infty}\left(\left[p_{0}, \bar{p}\right]\right)}+\frac{\eta}{2} \delta^{2} .
$$

The right-hand side of $(2.46)$ is $\geq f_{1}^{*}\left(p_{1}\right)$ as soon as $\delta \geq\left(2 \eta^{-1}\left\|f_{1}^{*}-f_{2}^{*}\right\|_{\mathbf{L}^{\infty}}\right)^{1 / 2}$. If (2.45) holds, we thus have

$$
p_{2} \leq p_{1}+\left|f_{1}^{\prime}(0)-f_{2}^{\prime}(0)\right|+\sqrt{\frac{2}{\eta}\left\|f_{1}^{*}-f_{2}^{*}\right\|_{\mathbf{L}^{\infty}\left(\left[p_{0}, \bar{p}\right]\right)}} .
$$

In conclusion, we obtain the estimate

$$
\left\|\left(f_{1}^{*}\right)^{-1}-\left(f_{2}^{*}\right)^{-1}\right\|_{\mathbf{L}^{\infty}([0, \kappa])} \leq C_{0} \cdot \sqrt{\left\|v_{1}-v_{2}\right\|_{\mathbf{L}^{\infty}([0, \bar{\rho}])}}
$$

for a suitable constant $C_{0}$. In the setting of Theorem 3 , the difference (2.34) between the starting distributions in the Nash equilibria with velocity functions $v_{1}, v_{2}$ can now be estimated by

$$
\left\|Q_{1}-Q_{2}\right\|_{\mathbf{L}^{1}([a, b])} \leq C \cdot \sqrt{\left\|v_{1}-v_{2}\right\|_{\mathbf{L}^{\infty}([0, \bar{\rho}])}},
$$

for a suitable constant $C$. 
3. A minimax property of Nash equilibria. Fix $\kappa>0$ and let $\mathcal{Q}^{\kappa}$ be the family of all left continuous, nondecreasing functions satisfying (1.9). For $Q \in \mathcal{Q}^{\kappa}$, recalling (1.12) define the functional

$$
\Psi(Q) \doteq \operatorname{ess}_{\beta \in[0, \kappa]}\left\{\varphi\left(x^{q}(\beta)\right)+\psi\left(x^{a}(\beta)\right)\right\}
$$

The next result shows that the Nash equilibrium provides a global minimizer to the functional $\Psi$.

Theorem 4. If $Q^{*}$ is the initial distribution of the Nash equilibrium solution with a number $\kappa>0$ of drivers, then

$$
\Psi\left(Q^{*}\right)=\min \left\{\Psi(Q): Q \in \mathcal{Q}^{\kappa}\right\}
$$

Proof. Let $\mathcal{Q}^{*} \in \mathcal{Q}^{\kappa}$ be the departure distribution for the Nash equilibrium solution with $\kappa>0$ drivers. Let $c=\Psi\left(Q^{*}\right)$ be the common total cost payed by each driver, in the Nash solution. Call $\mathcal{Q}_{c}$ the family of all departure distributions (with an arbitrary number of drivers), where each driver pays a total cost $\leq c$. As proved in [1, the Nash solution can be characterized as

$$
Q^{*}(x)=\sup \left\{Q(x): Q \in \mathcal{Q}_{c}\right\}
$$

To prove (3.2) assume that, on the contrary, there exists another departure distribution $Q^{-} \in \mathcal{Q}^{\kappa} \cap \mathcal{Q}_{c-\varepsilon}$, for some $\varepsilon>0$. By the analysis in [1], we can then construct a second distribution $Q^{+} \in \mathcal{Q}^{\kappa+\delta} \cap \mathcal{Q}_{c}$, for some $\delta>0$. But this provides a contradiction with (3.3), because

$$
\kappa=\lim _{x \rightarrow+\infty} Q^{*}(x)<\lim _{x \rightarrow+\infty} Q^{+}(x)=\kappa+\delta .
$$

REMARK 3. In general $Q^{*}$ is not the unique global minimizer for the functional $\Psi$. For example, recalling (1.11), consider the initial data

$$
\widetilde{Q}(x) \doteq U^{*}(0+, x)=\min _{y}\left\{Q^{*}(y)+M(x-y): y \leq x\right\} \leq Q^{*}(x)
$$

Then the corresponding solution $\widetilde{U}$ satisfies $\widetilde{U}(t, x)=U^{*}(t, x)$ for all $t>0$ and $x \in \mathbb{R}$. In particular, for any $\beta \in[0, \kappa]$, the departure and arrival times of the $\beta$-driver satisfy

$$
\tilde{x}^{q}(\beta)=x_{*}^{d}(\beta) \geq x_{*}^{q}(\beta), \quad \tilde{x}^{a}(\beta)=x_{*}^{a}(\beta)
$$

Hence

$$
\varphi\left(\tilde{x}^{q}(\beta)\right)+\psi\left(\tilde{x}^{a}(\beta)\right) \leq \varphi\left(x_{*}^{q}(\beta)\right)+\psi\left(x_{*}^{a}(\beta)\right)=c \doteq \Psi\left(Q^{*}\right)
$$


showing that $\widetilde{Q} \in \mathcal{Q}^{\kappa} \cap \mathcal{Q}_{c}$ also provides a minimizer for the functional $\Psi$. It is quite possible that $\widetilde{Q} \neq Q^{*}$. This happens when, in the Nash solution, some of the drivers wait in the queue. By allowing these drivers to depart exactly at time $\tilde{x}^{q}(\beta)=x_{*}^{d}(\beta)$, they avoid waiting in line, and hence pay a total cost strictly smaller than $c$.

4. Dynamic stability of Nash equilibria. A natural modeling assumption is that Nash equilibria should describe actual traffic patterns. However, a rigorous justification of this claim requires a nontrivial stability analysis. Indeed, assume that on a given day the drivers start their journey at times described by the distribution $Q(\cdot)$. If this is not a Nash equilibrium, on the next day some drivers may decide to change their schedule, starting at a different time, hopefully yielding a lower individual cost. We can thus consider a transformation $\mathcal{T}: Q(\cdot) \mapsto \widetilde{Q}(\cdot)$, describing how the distribution of departure times varies from one day to the next. For some meaningful choice of $\mathcal{T}$ we expect that, for any given initial distribution $Q(\cdot)$, the limit of iterates $\lim _{n \rightarrow \infty} \mathcal{T}^{n} Q$ should yield the unique Nash equilibrium solution.

Rather than a discrete transformation, we propose here two evolution equations for the distribution $Q(\cdot)$, depending on a continuous parameter $s$. Assume that the departure rate is absolutely continuous w.r.t. Lebesgue measure, so that

$$
Q_{x}(x)=\bar{u}(x)
$$

Let $U=U(t, x)$ be the corresponding solution of the Hamilton-Jacobi equation (1.8) computed by the Lax formula (1.10). Moreover, let

$$
u(t, x)=U_{x}(t, x) .
$$

For each $x \in \mathbb{R}$, call $y(x)$ the arrival time of a driver departing at time $x$. This is implicitly defined by

$$
Q(x)=U(T, y(x))
$$

We also define

$$
\Phi(x) \doteq \varphi(v x)+\psi(y(x))
$$

the total cost to a driver starting at time $x$. It is important to keep in mind that the map $x \mapsto y(x)$, and hence $\Phi(\cdot)$, depends on the overall traffic distribution, i.e. on $u$.

Next, consider a one-parameter family of initial data: $\bar{u}(x, s)=Q_{x}(x, s)$, depending on the auxiliary parameter $s \in \mathbb{R}_{+}$. We seek an evolution equation of the form

$$
\frac{d}{d s} \bar{u}=\Upsilon(\bar{u})
$$

for some (possibly nonlocal) functional $\Upsilon$, describing how drivers may change their departure time, in an attempt to reduce their total cost. Two choices for the right-hand side of (4.3) seem natural. 
Model 1. Drivers who initially depart at $x$ continuously modify their departure time, forward or backward depending on the gradient $\Phi_{x}(x)$ of the cost. In this case we can take $\Upsilon(\bar{u})=\left(\Phi_{x} \bar{u}\right)_{x}$ and the evolution of the starting distribution $\bar{u}=\bar{u}(x, s)$ is described by the conservation law

$$
\bar{u}_{s}-\left(\Phi_{x} \bar{u}\right)_{x}=0
$$

Model 2. Drivers who depart at time $x$ may decide to jump to a different departure time $\xi \in \mathbb{R}$, with probability proportional to the difference in cost. This leads to the integro-differential evolution equation

$$
\frac{d}{d s} \bar{u}(x)=\int \bar{u}(\xi)[\Phi(\xi)-\Phi(x)]_{+} d \xi-\int \bar{u}(x)[\Phi(x)-\Phi(\xi)]_{+} d \xi
$$

In both cases the key issue is whether, as $s \rightarrow+\infty$, the distribution with density $\bar{u}(\cdot, s)$ converges to the initial data of the Nash equilibrium solution.

4.1. Numerical simulations. A numerical computation of the Nash equilibrium can be achieved through the necessary condition of the optimal control problem. Let $c$ be the cost for every driver in the Nash solution. We discretize the Lagrangian variable $\beta$ identifying a particular driver, and set $\beta_{i}=i \cdot \delta$, for a fixed mesh $\delta>0$. The departure time $x_{i}^{q}=x^{q}\left(\beta_{i}\right)$ and the arrival time $x_{i}^{a}=x^{a}\left(\beta_{i}\right)$ of the $i$-th driver are computed by the following procedure.

- The first car finds no traffic ahead and travels with maximum speed $v(0)$. The total time needed for the trip is $\mu$, as defined in (1.14). The departure time $x_{1}^{q}$ of the first car is the smallest root of the equation

$$
\varphi(x)+\psi(x+\mu)=c .
$$

This can be found by a secant iterative method. We set

$$
x_{1}^{q}=t_{1}, \quad x_{1}^{a}=t_{1}+\mu .
$$

The largest root of the equation (4.6) provides an upper bound for the departure time of the last car.

- For $i \geq 2$, the times $x_{i}^{q}$ (when the $\beta_{i}$-driver begins his journey, possibly joining the queue) are computed in a sequential way. Given $x_{j}^{q}$ for all $j<i$, we determine $x_{i}^{q} \in\left[x_{i-1}^{q}, \infty[\right.$ as the smaller root of the equation

$$
\varphi(x)+\psi\left(x_{i}^{a}(x)\right)=c .
$$

Here

$$
\begin{aligned}
x_{i}^{a}(x) & =\min _{x \geq x_{i-1}^{q}}\left\{x+\mu, \sup _{j<i}\left\{x^{q}\left(\beta_{j}\right)-h^{-1}\left(\beta_{j}-\beta_{i}\right)\right\}\right\} \\
& =\min _{x \geq x_{i-1}^{q}}\left\{x+\mu, \sup _{j<i}\left\{x_{j}^{q}-h^{-1}((j-i) \delta)\right\}\right.
\end{aligned}
$$


is the arrival time of the $\beta_{i}$-driver if he departs at time $x$. This root of (4.7) can be efficiently computed by secant iteration, starting with the two initial guesses $x=x_{i-1}^{q}, x=x_{i-2}^{q}$. It is found that 2 to 3 iterations of the secant method suffice.

- If for $i=N+1$ no solution of (4.7) is found, then the iteration stops. The value $\kappa(c)=\beta_{N}=N \delta$ is the total number of cars in the Nash equilibrium solution with common cost $c$ to all drivers.

In a more realistic situation, instead of the cost $c$, one is given the total number of cars $\bar{\kappa}$. In this case, to compute the corresponding Nash solution one needs to invert the map $c \mapsto \kappa(c)$ and find a cost $\bar{c}$ such that $\kappa(\bar{c})=\bar{\kappa}$. This can be done numerically, again using secant iterations. For this purpose, it is found that 4 to 5 iterations suffice.
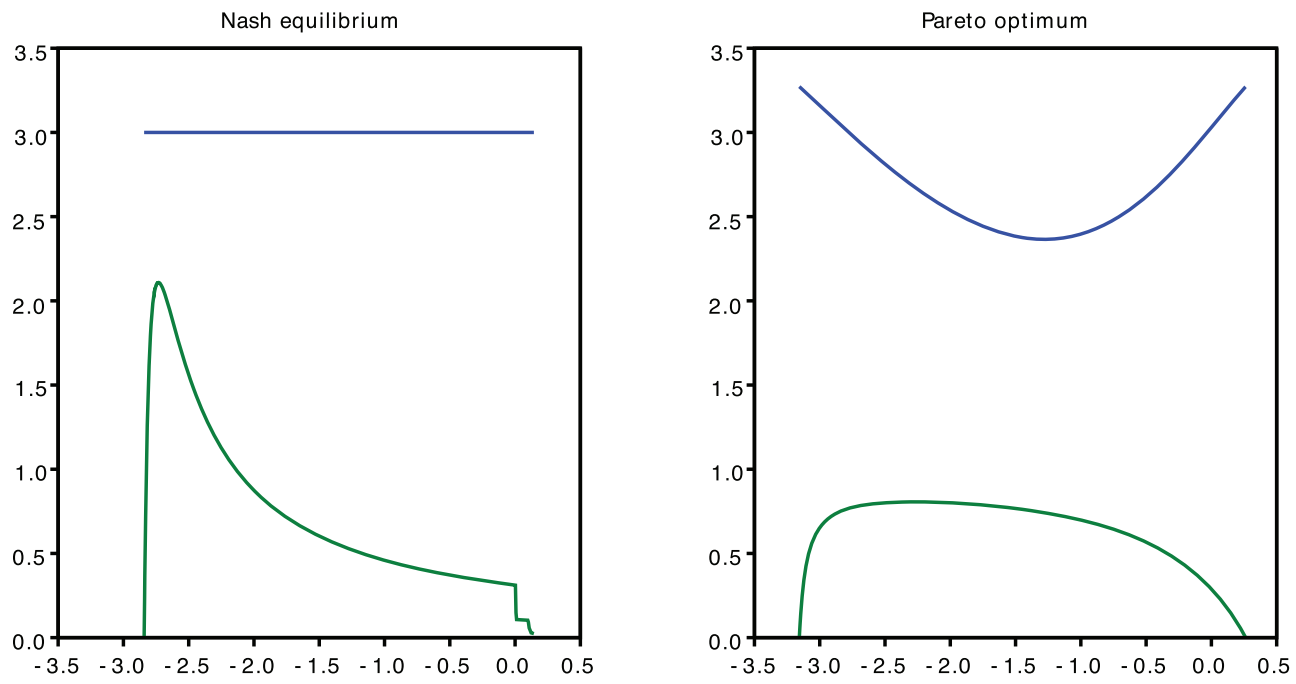

FIG. 6. Numerical solutions for the model (4.9). The total (departure + arrival) cost $\Phi$ (blue) and the rate of departures $\bar{u}$ (green) are plotted as functions of departure time $x$, for the Nash equilibrium solution and for the Pareto optimal solution. In both cases, the total number of cars is 2.2005 .

In our numerical simulations we consider a highway of length $l=2$. The velocity function in (1.2) and the departure and arrival costs in (1.1) are taken to be

$$
v(\rho)=2-\rho, \quad \varphi(x)=-x, \quad \psi(x)=e^{x},
$$

respectively. Figure [6] shows the rate $\bar{u}(x)$ at which cars depart (possibly joining the queue) and the total cost $\Psi(x)$ as functions of the departure time. The plots on the left refer to the Nash equilibrium solution, while the plots on the right yield the Pareto 
optimal solution, where the sum of the costs to all drivers is globally minimized. A detailed analysis of this globally optimal solution can be found in [1].

Simulations of the two dynamic models. To simulate Model 1, the conservation law (4.3) is solved by a Lax-Friedrichs scheme, satisfying the CFL conditions. To compute the flux $\Phi_{x}^{u}$, we apply a smooth filter (cubic spline) to guarantee that $\Phi$ is in $\mathcal{C}^{2}$. This eliminates numerical oscillations. The presence of this filter corresponds to a numerical diffusion term, leading to a more stable dynamical system.

To simulate Model 2, the trapezoid rule is used to numerically compute the integral on the right-hand side of (4.5). The resulting evolution equation is solved with a forward Euler scheme. The time step is chosen small enough so that $\bar{u} \geq 0$ for all $s$.

In both cases, we take the Pareto optimal solution as the initial data. As $s$ becomes larger, the profile $\bar{u}(\cdot, s)$ gets somewhat closer to the departure rate for the Nash equilibrium. However, numerical data do not provide evidence of asymptotic convergence as $s \rightarrow+\infty$.
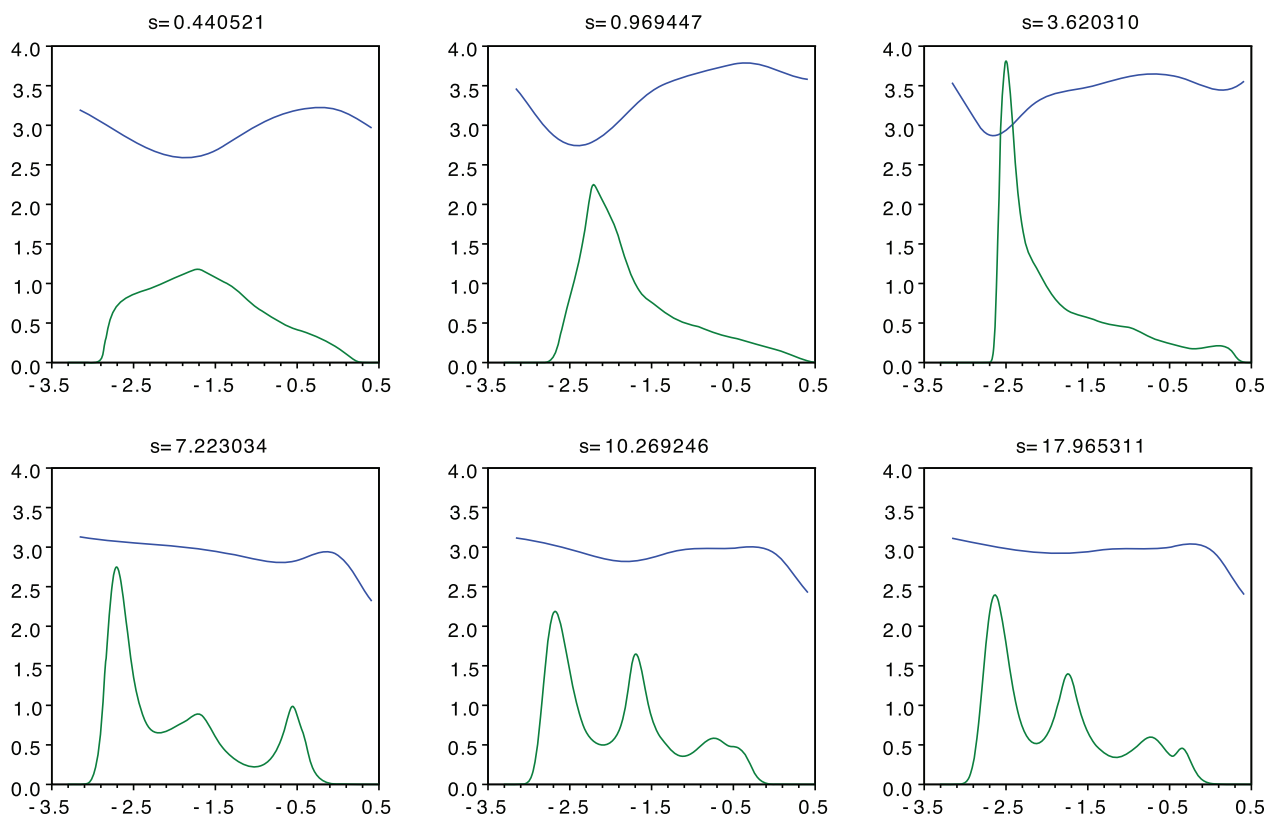

FIG. 7. Numerical simulations for Model 1. Here the total cost $\Phi$ and the rate of departures $\bar{u}$ are plotted as functions of the departure time $x$, for increasing values of $s$.

REMARK. A rigorous analysis of the models (4.4)-(4.5) is difficult, because in both cases the evolution operator is nonlocal. Even the linearized stability of these evolution equations, at the Nash equilibrium, remains an open problem. 

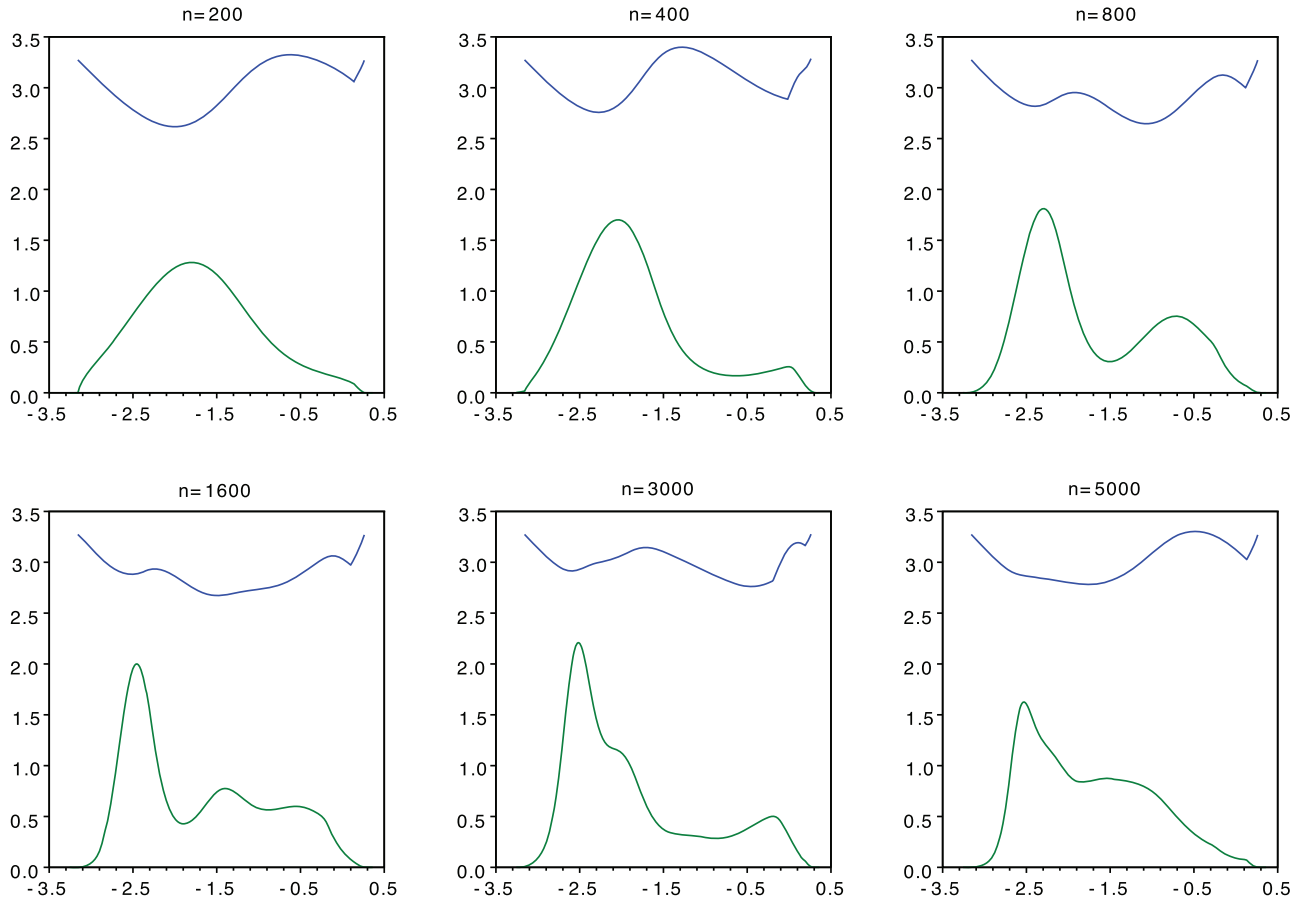

FIG. 8. Numerical simulations for Model 2. The total cost $\Phi$ and the rate of departures $\bar{u}$ are plotted as functions of the departure time $x$. Here the time step is taken to be $\Delta s=0.005$; hence a plot after $n$ steps shows a solution of 4.5) at time $s_{n}=0.005 \cdot n$.

Acknowledgements. The research of A. Bressan was partially supported by the NSF through grant EFRI-1024707 "A theory of complex transportation network design". C. Liu and F. Yu were both supported by the NNSF of China with grants 10971134 and 11031001, and by the Shanghai Jiao Tong University Innovation Fund for Postgraduates.

\section{REFERENCES}

[1] A. Bressan and K. Han, Optima and equilibria for a model of traffic flow. SIAM J. Math. Anal. 43 (2011), 2384-2417.

[2] T. L. Friesz, T. Kim, C. Kwon, and M. A. Rigdon, Approximate network loading and dual-time-scale dynamic user equilibrium. Transportation Research Part B (2010).

[3] A. Fügenschuh, M. Herty, and A. Martin, Combinatorial and continuous models for the optimization of traffic flows on networks. SIAM J. Optim. 16 (2006), 1155-1176. MR2219137 (2007k:90009)

[4] M. Garavello and B. Piccoli, Traffic Flow on Networks. Conservation Laws Models. AIMS Series on Applied Mathematics, American Institute of Mathematical Sciences, Springfield, Mo., 2006. MR.2328174 (2008g:90023)

[5] M. Gugat, M. Herty, A. Klar, and G. Leugering, Optimal control for traffic flow networks. J. Optim. Theory Appl. 126 (2005), 589-616. MR2164806 (2006m:49043)

[6] M. Herty, C. Kirchner, and A. Klar, Instantaneous control for traffic flow. Math. Methods Appl. Sci. 30 (2007), 153-169. MR2285119(2007k:93074)

[7] L. C. Evans, Partial Differential Equations. Second edition. American Mathematical Society, Providence, RI, 2010. MR2597943 (2011c:35002)

[8] P. D. Lax, Hyperbolic systems of conservation laws II. Comm. Pure Appl. Math. 10 (1957), 537-566. MR0093653(20:176) 
[9] M. Lighthill and G. Whitham, On kinematic waves. II. A theory of traffic flow on long crowded roads. Proceedings of the Royal Society of London: Series A, 229 (1955), 317-345. MR0072606 $(17: 310 \mathrm{a})$

[10] J. Smoller, Shock waves and reaction-diffusion equations. Second edition. Springer-Verlag, New York, 1994. MR.1301779 (95g:35002) 